\title{
Belphégor
}

Littérature populaire et culture médiatique

10-3 | 2011

Peter Pan

\section{Fictions populaires. Études réunies par Nicolas Cremona, Bernard Gendrel et Patrick Moran}

\section{Vittorio Frigerio}

\section{(2) OpenEdition}

1 Journals

\section{Édition électronique}

URL : http://journals.openedition.org/belphegor/408

DOI : 10.4000/belphegor.408

ISSN : 1499-7185

Éditeur

LPCM

\section{Édition imprimée}

Date de publication : 10 janvier 2011

\section{Référence électronique}

Vittorio Frigerio, «Fictions populaires. Études réunies par Nicolas Cremona, Bernard Gendrel et Patrick Moran », Belphégor [En ligne], 10-3 | 2011, mis en ligne le 10 janvier 2013, consulté le 22 septembre 2020. URL : http://journals.openedition.org/belphegor/408 ; DOI : https://doi.org/10.4000/belphegor. 408

Ce document a été généré automatiquement le 22 septembre 2020

\section{(c)}

Belphégor est mis à disposition selon les termes de la Licence Creative Commons Attribution - Pas d'Utilisation Commerciale - Pas de Modification 4.0 International. 


\title{
Fictions populaires. Études réunies par Nicolas Cremona, Bernard Gendrel et Patrick Moran
}

\author{
Vittorio Frigerio
}

\section{RÉFÉRENCE}

Nicolas Cremona, Bernard Gendrel et Patrick Moran (eds.), Fictions populaires, Paris,

Classiques Garnier, 250 p., ISBN : 978-2-905725-37-0

1 Ce recueil présente une variété d'études qui tentent d'identifier la présence du «populaire » à des époques diverses. Dans leur introduction signée à trois mains, les responsables de l'ouvrage commencent par spécifier que la différence entre une œuvre littéraire et une œuvre populaire est " plus une question d'institutionnalisation que de valeur intrinsèque » et que la "porosité des frontières » (p.11) entre les deux permet bien des passages et des transitions, parfois peu évidents de prime abord. Ils nuancent néanmoins cette prise de position initiale en spécifiant que « définir le populaire par les pratiques d'édition, de lecture et de diffusion qui l'entourent ne saurait être un cadre suffisant à sa compréhension » (p. 18). Les méthodes d'écriture, les structures internes, les «caractéristiques formelles" (p. 20) jouent aussi un rôle important pour arriver à identifier des corpus d'ouvrages possédant des qualités susceptibles de pouvoir les assembler sous une catégorie donnée - c'est-à-dire, finalement, et toute "porosité " éventuelle rangée au deuxième plan - à l'intérieur ou à l'extérieur de la sphère littéraire (aussi vague que l'on veuille bien malgré tout garder ce concept). La gageure du recueil est de montrer que même en dehors du xixe siècle, si largement défriché par les chercheurs, il existe des productions romanesques, ou du moins fictionnelles, qui présentent des formes narratives essentiellement semblables à celles qui marqueront le début de l'époque de la « littérature de masse ». Dans leur intention, la leçon à tirer de cet ensemble d'études allant du Moyen Âge au présent est que «l'appartenance à la catégorie du populaire ou du non-populaire (si ces catégories ont encore un sens, au 
terme de notre analyse) ne signifie rien d'autre qu'un certain contrat de lecture, qu'une certaine organisation des éléments constitutifs du texte » (p. 24).

2 Il se trouve cependant que toutes les études présentées ici ne pointent guère de manière aussi univoque vers une conclusion à ce point ouverte et œcuménique.

3 L'ouvrage est divisé en quatre parties. La première, "Réflexions sur la notion de "fiction populaire" ", réunit des textes de Jacques Migozzi, Denis Labouret et Raphaël Baroni, de nature essentiellement théorique. La deuxième («Fonctionnement et réception du populaire ») présente des travaux de Lise Andries, Nicola Cremona, Camille Wolff, Daniel Compère et Irène Langlet, allant du Moyen Âge au XIVI ${ }^{e}$ siècle, pour sauter ensuite au XIx et à la science-fiction contemporaine. La troisième partie («Parcours du populaire») est consacrée encore au Moyen Âge (Emmanuelle PoulainGautret, Patrick Moran) au roman gothique (Daniel Couégnas) et au roman de mœurs (Bernard Gendrel), les deux dernières contributions (Jean-Pierre Aubrit et JeanChristophe Blum) traitent respectivement du mythe de Cyrano et des séries télévisées Buffy et Lost. L'arc temporel va donc des tout débuts de la création littéraire identifiable en tant que telle à l'extrême contemporain médiatique.

Une grande partie de l'intérêt, et en même temps des limites, du recueil peut s'identifier à partir de la déclaration d'intention dans l'article de Patrick Moran, «Cycles, séries et écriture épisodique : l'exemple du Moyen Âge » :

La recherche en paralittérature, tout en s'intéressant de près à la réalité éditoriale et historique de la littérature "populaire » ou « de grande consommation », a aussi eu à cœur d'étudier les critères formels qui distinguent le paralittéraire du littéraire et d'élaborer un modèle paralittéraire, pour reprendre les termes de Daniel Couégnas. Or l'existence même de ces modèles théoriques permet d'élargir le champ des recherches : peut-être des traits du modèle paralittéraire se retrouventil, en l'état ou sous un aspect légèrement différent, à des époques antérieures (p. 163).

Or, le désavantage de l' " originalité » de cette approche - qui est en fait l'extension des études «paralittéraires » telles qu'elles se pratiquaient il y a une vingtaine d'années à des périodes encore peu fréquentées jusqu'ici par les amateurs du genre, fait qu'il y a parfois pour les habitués un très fort sentiment de déjà-vu quand on analyse les œuvres avec des schémas extrêmement connus des dix-neuviémistes. La méthode tend à dicter les conclusions et la seule surprise, si de surprise il s'agit, est qu'effectivement, un air de famille indéniable, quoique généralement sous-estimé, réunit des œuvres d'époques fort éloignées. De plus, le recensement de nombre de critères formels auquel s'adonnent plusieurs participants au recueil contredit les conclusions posées par Jacques Migozzi en début du volume ("Cet obscur objet du désir universitaire. Coup d'œil dans le rétroviseur sur 15 ans de recherches sur les fictions populaires »), stipulant qu' "[i]l n'est donc plus possible aujourd'hui au chercheur littéraire de s'en tenir à la recherche de critères formels propres à la fiction populaire, ou plutôt de croire que ce recensement poéticien suffirait à comprendre l'essentiel de ce qui se joue dans le tête a tête intime du lecteur et de la fabula qu'il s'approprie» (p.43). Des approches d'essence narratologique, où reviennent les noms de Genette, de Todorov, d'Eco, et les analyses proposées par Daniel Couégnas dans son Introduction à la paralittérature, donnent ainsi parfois l'impression d'être revenus en arrière, au grand minimum, des 15 ans qui font l'objet du regard rétrospectif de Migozzi. Il est d'ailleurs intéressant, si ce n'est révélateur, de voir comment Daniel Couégnas lui-même, dans sa contribution («Circulation, permanence et plasticité des formes littéraires. Des 
Mystères d'Udolpho aux fictions populaires ») n'utilise jamais le terme paralittérature, préférant "fiction "populaire" ou "de grande consommation" » (p. 191). Sa conclusion mérite d'être soulignée: "parler de fiction populaire, c'est pour nous, depuis très longtemps parler de la littérature, dans son unité et sa diversité » (p. 192).

Ceci dit, et ces contradictions constitutives mises à part, le recueil réserve de bons moments de lecture et de belles découvertes. Dans son article «Derrière le fiacre de Fantômas ou l'ascenseur d'Arsène Lupin: l'aventure encore et toujours?", Denis Labouret retrace les cheminements de l'aventure du côté de Gracq, Malraux et Sartre, y retrouvant « une force non une forme du récit» (p. 62), faisant d'elle un lieu privilégié entre romans populaires et «intellectuels». Raphaël Baroni («L'intrigue est-elle populaire?») offre des pistes de réflexion intrigantes (le mot s'impose) en suggérant "l'hypothèse que la différence entre une mise en intrigue fondée sur la défamiliarisation du quotidien et une mise en intrigue dépendant d'événements dont la saillance est marquée socialement pourrait être un critère de distinction entre les romans modernes canonisés par la critique et les romans dits "populaires" » (p. 79). Lise Andries ( Les romans de chevalerie de la bibliothèque bleue ») montre comment le discours bien connu des modernes sur la vitalité de la littérature populaire "qui pourrait régénérer une narrativité contemporaine exsangue» (p.96) date déjà du temps de la Bibliothèque bleue. Nicolas Cremona («Les histoires tragiques, fictions populaires?») explore les similitudes et les différences entre le " genre » des Histoires tragiques (ne pouvant pas parler de "paralittérature » en dehors du contexte sociohistorique du xix ${ }^{e}$ siècle) et les canards. Camille Wolff (« Rééditions contemporaines de littérature populaire ») s'interroge sur le processus de réédition d'une littérature qu'on voudrait « jetable », discutant du phénomène des collections, du discours promotionnel qui entoure les œuvres et le nom de l'écrivain, et des stratégies de fidélisation des lecteurs. Daniel Compère propose, comme l'indique son sous-titre, «Quelques pistes de réflexion" sur le thème de "Popularité et pérennité " à travers la discussion de la «fortune » de Dumas, Ponson et Aimard. Irène Langlet (« Au ras des planètes : quelle poétique des science-fictions populaires?») offre une définition pratique de la science fiction (il s'agirait d'une "narration merveilleuse et aventureuse argumentable » p. 136) avant de disserter sur le clivage entre l'époque Gernsback et l'époque Campbell et de retrouver dans la production science-fictionnelle un schéma tripartite qui évoque la hiérarchisation highbrow/middlebrow/lowbrow de MacDonald. Emmanuelle PoulainGautret («Renart est-il un héros de littérature populaire?») s'interroge sur la survie de ce héros négatif et ambigu, et sur la multiplication de ses aventures, ainsi que sur l'existence dans ces textes d'une culture faite de références savantes mélangée à des structures proches de celles du roman populaire. Patrick Moran («Cycles, séries et écriture épisodique: l'exemple du Moyen Âge ») identifie dans le Cycle Vulgate la naissance d'un type de narration à épisodes en trouvant entre littérature arthurienne et "paralittérature » un air de famille fait de répétition, stéréotypie et monologisme, qui «ne sont pas des critères dépréciatifs dans le contexte médiéval» (p. 171). Daniel Couégnas ( Circulation, permanence et plasticité des formes littéraires. Des Mystères d'Udolpho aux fictions populaires »), part de la littérature gothique et passe par les «romanciers et poètes romantiques, Balzac, Hugo, les sœurs Brontë, écrivains fantastiques comme Poe ou Stevenson, Surréalistes » pour " apporter très brièvement sa contribution à l'idée de circulation permanente des thèmes et des formes entre les registres, du plus élitiste au plus populaire » (p. 188). Bernard Gendrel (« Le "roman de mœurs", roman populaire ou roman réaliste ?»), pose une hypothèse de base : «que le 
genre du "roman de mœurs", en lui-même, n'est ni populaire ni réaliste, mais qu'il possède des caractéristiques qui peuvent le conduire dans ces deux voies concurrentes» (p. 200). Jean-Pierre Aubrit (« De Savinien à Cyrano : la fabrication d'un héros populaire»), retrace l'évolution du personnage historique, au mythe, à son adoption par l'imaginaire populaire. Enfin, Jean-Christophe Blum («L'arc et le retour : pour une poétique des séries télévisées ») retrouve dans les histoires de vampires de Buffy et le monde perdu de Lost « la vieille structure formule, essentiellement musicale, du thème et de ses variations » (p. 229).

7 Si le but des responsables de l'ouvrage était bien, comme l'indique la quatrième de couverture, d'identifier certains "mécanismes essentiels de l'écriture romanesque " bien avant l'explosion de la littérature de masse au XIX ${ }^{\mathrm{e}}$ siècle, il faut considérer leur entreprise comme une réussite en dépit des variations de qualité et d'approfondissement qu'il peut y avoir dans les études ici réunies. On peut regretter cependant que la solution de facilité d'assimiler l'écriture "populaire » aux « critères formels» qui la constitueraient ait conduit certains chercheurs, familiers de périodes bien antérieures à celle de la naissance de la culture de masse, à des exercices quelque peu mécaniques qui ne font pas vraiment regretter les temps encore assez proches quand narratologie et structuralisme dominaient entièrement l'horizon critique. 